\title{
SVT discrimination algorithms significantly reduce the rate of inappropriate therapy in the setting of modern day delayed high-rate detection programming
}

\author{
Cheng, Alan ; Auricchio, Angelo ; Schloss, Edward J ; Kurita, Takashi ; Sterns, Laurence D ; Gerritse,
} Bart ; Brown, Mark L ; Fagan, Dedra H ; Lexcen, Daniel R ; Ellenbogen, Kenneth A

\begin{abstract}
BACKGROUND Contemporary ICD programming involving delayed high-rate detection and use of SVT discriminators has significantly reduced the rate of inappropriate shocks. The extent to which SVT algorithms alone reduce inappropriate therapies is poorly understood. METHODS AND RESULTS PainFree SST enrolled 2,770 patients with a single or dual-chamber ICD or cardiac resynchronization defibrillator. Patients were followed for $22 \pm 9$ months with SVT discriminators on in $96 \%$ of patients. Sustained ventricular tachyarrhythmias and SVT episodes were adjudicated by an independent physician committee. For this analysis, all episodes were subjected to post-processing computer simulation with SVT discriminators off with and without delayed high-rate detection criteria (VF zone only, 30/40@320ms). There were 3,282 adjudicated SVT episodes of which 115 resulted in an ICD shock and 113 received only ATP (2-year inappropriate shock and therapy rates of $3.1 \%$ and $4.1 \%$ ). Therapy was appropriately withheld for the remaining 3,054 SVT episodes. With both SVT discriminators and delayed high-rate detection simulated off, the 2-year inappropriate therapy rate would have been $22.9 \%$ (Hazard Ratio $[\mathrm{HR}]=6.24,95 \%$ confidence interval $[\mathrm{CI}]: 5.20-7.49)$. With SVT discriminators simulated off and delayed high-rate detection simulated on in all patients, the 2-year rate would have been $6.4 \%(\mathrm{HR}=1.63$, CI: 1.44-1.85). CONCLUSIONS Use of SVT discriminators has a significant role in reducing the rate of inappropriate ICD therapy even in the setting of delayed high-rate detection settings. Deactivating SVT discriminators would have resulted in an overall increase in the inappropriate ICD therapy rate by $63 \%$ and $524 \%$ with and without delayed high-rate detection programming, respectively. This article is protected by copyright. All rights reserved.
\end{abstract}

DOI: https://doi.org/10.1111/jce.14250

Posted at the Zurich Open Repository and Archive, University of Zurich

ZORA URL: https://doi.org/10.5167/uzh-176410

Journal Article

Accepted Version

Originally published at:

Cheng, Alan; Auricchio, Angelo; Schloss, Edward J; Kurita, Takashi; Sterns, Laurence D; Gerritse, Bart; Brown, Mark L; Fagan, Dedra H; Lexcen, Daniel R; Ellenbogen, Kenneth A (2019). SVT discrimination algorithms significantly reduce the rate of inappropriate therapy in the setting of modern day delayed high-rate detection programming. Journal of cardiovascular electrophysiology, 30(12):2877-2884.

DOI: https://doi.org/10.1111/jce.14250 
Alan Cheng ORCID iD: 0000-0002-8213-9115

Dedra Fagan ORCID iD: 0000-0002-1430-4588

\title{
SVT discrimination algorithms significantly reduce the rate of inappropriate therapy in the setting of modern day delayed high-rate detection programming
}

\author{
Alan Cheng, $\mathrm{MD}^{1}$, Angelo Auricchio, MD, $\mathrm{PhD}^{2}$; Edward J. Schloss, $\mathrm{MD}^{3}$; Takashi \\ Kurita, $\mathrm{MD}, \mathrm{PhD}^{4}$; Laurence D. Sterns, $\mathrm{MD}^{5}$; Bart Gerritse, $\mathrm{PhD}^{6}$, Mark L. Brown, $\mathrm{PhD}^{1}$, \\ Dedra H. Fagan, $\mathrm{PhD}^{1}$, Daniel R. Lexcen, $\mathrm{PhD}^{1}$, Kenneth A. Ellenbogen, $\mathrm{MD}^{7}$
}

Short title: SVT discriminators and inappropriate therapy

\begin{abstract}
${ }^{1}$ Medtronic, plc, Mounds View, Minnesota; ${ }^{2}$ Fondazione Cardiocentro Ticino, Lugano, Switzerland; ${ }^{3}$ The Christ Hospital-Ohio Heart \& Vascular, Cincinnati, Ohio; ${ }^{4}$ Kindai University School of Medicine, Osaka, Japan; ${ }^{5}$ Royal Jubilee Hospital, Victoria, Canada;

${ }^{6}$ Medtronic Bakken Research Center, Maastricht, The Netherlands; ${ }^{7}$ Virginia

Commonwealth University, Richmond, Virginia
\end{abstract}

\section{Address for Correspondence:}

Kenneth A. Ellenbogen, MD

Virginia Commonwealth University

Division of Cardiology 
Gateway Building, $3^{\text {rd }}$ Floor, 3-216

1200 East Marshall Street

Richmond, Virginia 23298-0053

Telephone: 804-828-7565; Fax: 804-826-6082

Email: kenneth.ellenbogen@vcuhealth.org

\begin{abstract}
Background: Contemporary ICD programming involving delayed high-rate detection and use of SVT discriminators has significantly reduced the rate of inappropriate shocks. The extent to which SVT algorithms alone reduce inappropriate therapies is poorly understood.
\end{abstract}

Methods and Results: PainFree SST enrolled 2,770 patients with a single or dualchamber ICD or cardiac resynchronization defibrillator. Patients were followed for $22 \pm 9$ months with SVT discriminators on in $96 \%$ of patients. Sustained ventricular tachyarrhythmias and SVT episodes were adjudicated by an independent physician committee. For this analysis, all episodes were subjected to post-processing computer simulation with SVT discriminators off with and without delayed high-rate detection criteria (VF zone only, 30/40@320ms). There were 3,282 adjudicated SVT episodes of which 115 resulted in an ICD shock and 113 received only ATP (2-year inappropriate shock and therapy rates of $3.1 \%$ and $4.1 \%$ ). Therapy was appropriately withheld for the remaining 3,054 SVT episodes. With both SVT discriminators and delayed high-rate

This article is protected by copyright. All rights reserved. 
detection simulated off, the 2-year inappropriate therapy rate would have been $22.9 \%$ (Hazard Ratio $[\mathrm{HR}]=6.24,95 \%$ confidence interval $[\mathrm{CI}]: 5.20-7.49)$. With SVT discriminators simulated off and delayed high-rate detection simulated on in all patients, the 2-year rate would have been $6.4 \%$ (HR=1.63, CI: 1.44-1.85).

Conclusions: Use of SVT discriminators has a significant role in reducing the rate of inappropriate ICD therapy even in the setting of delayed high-rate detection settings. Deactivating SVT discriminators would have resulted in an overall increase in the inappropriate ICD therapy rate by $63 \%$ and $524 \%$ with and without delayed high-rate detection programming, respectively.

Clinical Trial Registration: URL: http://clinicaltrials.gov Unique Identifier: NCT00982397

Keywords: Implantable cardioverter defibrillator, cardiac resynchronization therapy, heart failure, atrial fibrillation, inappropriate shock

\section{Introduction}

Early implantable cardioverter defibrillator (ICD) programming was designed to rapidly detect and treat ventricular arrhythmias. ${ }^{1}$ Unfortunately, this resulted in therapies that were either inappropriate or unnecessary. In particular, among patients receiving ICDs for primary prevention of sudden cardiac death, almost $40 \%$ of the therapies delivered were deemed inappropriate or unnecessary. Recent advancements in our understanding of arrhythmia outcomes and improvements in ICD diagnostics and algorithms have placed greater focus on treating longer, faster ventricular arrhythmias

through strategic device programming (i.e., delayed high-rate detection ${ }^{2,3,4}$ ),

This article is protected by copyright. All rights reserved. 
antitachycardia pacing (ATP) ${ }^{5}$ use, and/or the application of supraventricular tachycardia (SVT) discriminators. While the programming options are similar across the different device manufacturers, recent Heart Rhythm Society (HRS) guidance have cautioned against generalizing learnings from one manufacturer's device platform to others because operation details differ. ${ }^{6}$ Hence, there is a need to better understand patient outcomes from these interventions that are contemporary and device manufacturer-specific.

The PainFree SST trial was a prospectively enrolling study that included primary and secondary prevention Medtronic ICD recipients to assess the effectiveness of SmartShock Technology ${ }^{\mathrm{TM}}$ (SST), a suite of 6 proprietary SVT and non-arrhythmia discriminators (for rejection of lead noise, $\mathrm{T}$ wave oversensing, and lead integrity alert) aimed at reducing overall inappropriate ICD therapies ${ }^{7,8}$ in the setting of modern day use of delayed high-rate detection programming and ATP. This study demonstrated an overall inappropriate ICD shock rate of $1.5 \%$ at 12 months in dual and triple chamber ICDs and $2.5 \%$ in single chamber ICDs, the lowest reported in any large prospective trial. ${ }^{8,9}$ Whether the primary benefit was derived from delayed high-rate programming or from the use of SVT discriminators remains poorly understood. This analysis aims to better understand the individual contributions of these two strategies in reducing overall inappropriate therapies.

\section{Methods}

\section{Patients and Procedures}

The PainFree SST trial has been previously described. ${ }^{7,8}$ In brief, PainFree SST was a global, prospective, multicenter trial of patients undergoing de-novo or 
replacement implantation (based on current clinical practice guidelines) of an ICD or cardiac resynchronization defibrillator (CRT-D) with SST (ClinicalTrials.gov ID: NCT00982397). All detected episodes with at least 16 beats in the ventricular detection zones and stored with electrograms (EGMs), including ventricular arrhythmias, nonsustained ventricular tachycardia (NSVT) and SVT, were adjudicated by an independent physician episode adjudication committee (EAC) ${ }^{8}$ The ethics committee or IRB at each participating institution approved the study protocol and all subjects provided written informed consent.

\section{Device Programming}

Tachyarrhythmia detection and therapies were pre-specified by the study protocol to include a VF zone with number of intervals to detect (NID) at 30/40 for primary prevention patients and recommended detection interval of $320 \mathrm{msec}$. Secondary prevention patients were randomized between NID 18/24 and 30/40. ${ }^{10}$ No VT or fast VT (FVT) zone was recommended for primary prevention patients and programmed per physician discretion for secondary prevention patients. Additional specified programming in both groups included ATP therapy delivered during charging in the VF zone, and SVT discriminators on for a median ventricular interval $\geq 260 \mathrm{~ms}$. Deviations to these programmed parameters were made per physician discretion with medical justification. ${ }^{8}$

\section{Statistical Analysis}

Given the homogeneity in the way patients were programmed, computer-based simulations were performed to determine what the outcome (i.e., therapy delivered or withheld) for a given arrhythmia would have been if certain programming features were

This article is protected by copyright. All rights reserved. 
activated or deactivated. The scenarios are shown in Supplemental Table 1 and included (1) turning off all SVT and ventricular oversensing (VOS) discriminators, (2) having SVT/VOS discriminators off in the setting of delayed high-rate detection, and (3) having only pre-SmartShock Technology ${ }^{\mathrm{TM}}$ discriminators on. Figure 1 illustrates simulation scenarios (1) and (2). Panel A is the original AF episode with detection withheld by the SST algorithms. In scenario 1, this episode would have been inappropriately detected and treated. The episode would not have been detected in scenario 2. Incidence of ICD therapies was estimated using Cumulative Incidence Functions treating death as a competing risk. Cox proportional hazards regression was used to estimate the hazard ratio (HR) for incidence of therapy between simulated scenarios and actual observed therapy incidence, accounting for within-patient correlation by the use of a robust covariance estimated by the Generalized Estimating Equations (GEE) approach. ${ }^{11}$ GEE was also used in context of logistic regression to estimate success of first ATP therapy.

\section{Results}

Among 2,770 patients (single-chamber [27\%], dual-chamber [34\%], and CRT [39\%] defibrillators; 33\% replacements or upgrades), 2,599 had device programming information available for analysis. Patient characteristics were similar to previously published ICD studies (Table 1) and patients were followed for $22 \pm 9$ months. VF detection was programmed on in 2,591 patients (99.7\%). The NID was programmed to $30 / 40$ for 1,693 primary prevention patients (96\%) and 387 secondary prevention patients (48\%). In most of the remaining patients, the NID was programmed to 18/24 (69 primary prevention patients [4\%] and 422 secondary prevention patients [52\%]). VT detection and therapies were programmed on in $62 \%$ of patients with a median detection rate of 
350ms (primary prevention: $57 \%$, median rate: $340 \mathrm{~ms}$; secondary prevention: $72 \%$, median rate: $360 \mathrm{~ms})$. SmartShock Technology ${ }^{\mathrm{TM}}$ was programmed on in $96 \%$ of patients. The SVT limit was programmed to the nominal value of $260 \mathrm{~ms}$ in $98 \%$ of the patients (Table 2).

The EAC adjudicated all 9,660 episodes with available EGMs and with at least 16 beats in the programmed detection zones. Table 3 and Figure 2 present how the device classified the episodes in relation to the adjudicated true rhythm. For 804 episodes, one or more shocks were delivered. In total, 115 episodes had inappropriate shocks for an inappropriate shock rate of $1.8 \%$ at 1 year and $3.1 \%$ at 2 years post implant. Additionally, 113 episodes had inappropriate ATP therapy. The incidence of inappropriate therapy was $2.7 \%$ at 1 year and $4.1 \%$ at 2 years (Figure 3 , blue line).

Among the 9,660 episodes, 3,282 episodes that met the rate-based detection criteria were adjudicated as SVT, T-wave oversensing, or lead noise. Therapy was appropriately withheld for 3,054 episodes (93.1\%). Only 6.9\% received inappropriate therapy and only $3.5 \%$ received a shock.

A high percentage of shocks (58.7\%) were appropriately caused by monomorphic VT; $34.3 \%$ of these were faster than $260 \mathrm{~ms}$, the nominal SVT Limit, and only $50.8 \%$ received ATP as first therapy. Overall, there were 3,316 episodes appropriately treated with ATP as first therapy. This first ATP therapy was successful for 2,857 episodes (86.2\%). Restricted to episodes adjudicated as monomorphic VT, first ATP therapy was successful for 2,829 (86.5\%) out of 3,270 episodes that received ATP as first therapy.

This article is protected by copyright. All rights reserved. 
Corrected for multiple episodes per patient using GEE, first ATP therapy success was 82.5\% (95\% confidence interval [CI]: 79.5\%-85.2\%).

Since $96 \%$ of patients had SST discriminators on, we performed computersimulated arrhythmia detection as if SST was deactivated across all 3,282 SVT episodes to determine if therapy would have been delivered. The worst-case inappropriate therapy rate was simulated by assuming therapy delivery at the moment of detection for all 3,282 episodes - corresponding to programming all SVT/VOS discriminators off with all other detection settings including rate and duration programming unchanged (see Table 2). In total, 553 patients would have had one or more inappropriate therapies and the incidence would have been $17.6 \%$ at 1 year and $22.9 \%$ at 2 years (Figure 3, red line). Compared to the observed rate, the increase is $524 \%$ (HR $=6.24$, CI: 5.20-7.49, $\mathrm{p}<0.0001$ ). A second scenario that was simulated involved having SVT/VOS discriminators off but uniformly setting all detection parameters to mimic delayed high-rate detection where only a VF zone requiring $30 / 40$ beats to be faster than $320 \mathrm{~ms}$. This resulted in 480 SVT episodes among 158 patients receiving therapy. The incidence rate of inappropriate therapy would have been $4.4 \%$ at 1 year and $6.4 \%$ at 2 years (Figure 3, green line). Compared to the observed rate, the increase is $63 \%$ ( $\mathrm{HR}=1.63$, CI: $1.44-1.85, \mathrm{p}<0.0001)$. Simulating these same detection parameters of only a VF zone requiring 30/40 beats to be faster than 320ms, but with SVT/VOS discriminators on, the incidence rate of inappropriate therapy would have been $2.2 \%$ at 1 year and $3.3 \%$ at 2 years (Figure 3, black line; HR=0.814, CI: 0.740-0.895, $\mathrm{p}<0.0001)$.

Atrial fibrillation (AF) was the most common cause of inappropriate therapies and PainFree SST included 818 patients (30\%) with a history of AF. Excluding these patients, This article is protected by copyright. All rights reserved. 
the observed incidence of inappropriate therapy was $2.0 \%$ at 1 year and $3.2 \%$ at 2 years. With delayed high-rate detection, the incidence would have been $3.8 \%$ at 1 year and $5.6 \%$ at 2 years, which is an increase of $81 \%(\mathrm{HR}=1.81, \mathrm{CI}: 1.51-2.17, \mathrm{p}<0.0001)$.

The third simulation scenario involved having delayed high-rate detection with

only pre- SST discrimination. Dual chamber discrimination include PR Logic, onset and stability without wavelet. Single chamber discrimination included wavelet, onset and stability. All devices used SVT Limit=320 ms. Lead noise and TWOS discriminators were excluded from all devices. The incidence of inappropriate therapy would have been $4.2 \%$ at 1 year and $6.1 \%$ at 2 years.

In terms of VT zone programming, 59\% of dual chamber patients had a VT zone programmed on with shocks (median VT detection interval of 340ms and 350ms for single and dual chamber, respectively). Interestingly, there was no difference in the inappropriate shock rate between those with VT zone on vs off ${ }^{8}$, suggesting a role of the SST discriminators in providing substantial shock reduction benefit.

\section{Discussion}

Our aim was to examine the incremental benefit of SVT discriminators beyond only delayed high rate programming and the benefit of each component of the algorithm that contribute to overall reduction in inappropriate therapies that were observed in the PainFree SST trial. This analysis has several important findings: (1) the use of SVT and VOS discriminators combined with real-world programming results in a low rate of inappropriate ICD therapies among primary and secondary prevention ICD recipients; (2) with SST discriminators programmed off, but rate and duration unchanged in these

This article is protected by copyright. All rights reserved. 
patients, the inappropriate ICD therapy rate would have increased by 524\%; (3) with sustained high rate programming and SST discriminators programmed off, the inappropriate ICD therapy rate would have increased by $63 \%$; and (4) combined use of SVT and VOS discriminators with sustained high rate programming would have incrementally decreased the inappropriate ICD therapy rate by $18.6 \%$. Detailed analysis of the specific rhythm outcomes and several post hoc simulations of different programming scenarios demonstrate that these low inappropriate shock rates could not be achieved without the use of these new discriminators. While AF remained the primary cause of inappropriate shocks, the SST discriminators were $84 \%$ specific for identifying $\mathrm{AF}$ and accounted for a substantial part of the difference between discriminators on vs. off. Although we focused primarily on the reduction in inappropriate therapy, it is worth noting that ATP provided substantial benefit in reducing unnecessary shocks for monomorphic VT. Of 3,497 episodes of monomorphic VT receiving therapy, ATP was associated with the termination of 2,994 (89.1\%) episodes.

In a comprehensive review of studies over the past 15 years, $6.4 \%$ of single chamber ICD patients had received an inappropriate shock at 12 months. ${ }^{9}$ The PainFree SST trial stands out among the trials included in this review as having an unusually low inappropriate shock rate in spite of having broad inclusion criteria allowing patients with $\mathrm{AF}$ and device replacements to be enrolled. These results suggest that the SST discriminators played an important role in combination with modern programming practices. In general, rate programming in the PainFree SST trial was slower than guidelines in part because secondary prevention patients were included, but also due to physician discretion. While exercising physician discretion deviates from what would be 
considered state of the art programming, it does reflect real world practice when real patients are being treated. Among primary prevention patients, 58.6\% had a VT zone programmed with shock therapies. In spite of this, patients with VT shock programmed on had a comparable inappropriate shock rate to that of patients without VT shocks primarily because of the SVT discriminators. How well these findings apply to other proprietary discriminator algorithms is unclear given that direct comparisons of different algorithms are rarely performed. In one such study, algorithms from two device manufacturers were compared in a randomized trial demonstrating superiority of one over another. ${ }^{12}$ By the time the study was completed and results reported, the competing algorithms had been modified. Hence, caution should be exercised when generalizing the findings of this study to other types of discriminators. Perhaps what is more important is the recent HRS statement highlighting the differences between device manufacturers and to avoid generalizing results from one manufacturer's device to another. ${ }^{6}$ This accentuates the need for having manufacturer-specific studies such as this one so that customized recommendations can be developed to maximize patient outcomes (Appendix B). ${ }^{1}$

While SST has resulted in significant improvements in the rates of inappropriate therapies, there is still room for improvement. Enhancements made to improve specificity must be balanced by potential compromises on safety as measured by delayed detection or loss of consciousness. These were reported in the primary results manuscript of the PainFree SST trial. ${ }^{8}$ Detection sensitivity was $98.8 \%$. As seen in table 3 there were 2 polymorphic rhythms for which detection was withheld by SVT/VOS discriminators. In 
addition, there were 17 additional episodes of polymorphic rhythms with delayed detection due to SVT/VOS discriminators. None led to death or major adverse events. ${ }^{8}$

\section{Limitations}

Several limitations were present that may affect the ability to apply these findings to a larger population. First, this was not an interventional trial and the impact of SVT discriminators being turned off was assessed using computer-based simulations. However, these methodologies have been successfully used in prior studies. ${ }^{13}$ Second, the simulations did not factor in a delay that often would result from the ICD capacitor charging. We have assumed that with modern ATP programming (including during charge) therapy is delivered at time of detection. Additionally, we analyzed not just shocks but all types of therapy including ATP. The timing of ATP delivery would not have been affected by the lack of having a capacitor delay. Third, the stored RR intervals that were the basis for our simulation often do not cover the full duration of ongoing SVT episodes. The simulation of delayed high rate detection settings may therefore have underestimated the number of episodes that would have received therapy. There were at least 977 episodes that had no simulated therapy but where the available interval data was truncated. Lastly, the PainFree SST study was a prospective observation study for the primary prevention patients and a randomized study for the secondary patients. All of the potential biases associated with the primary prevention prospective arm of the study affecting PainFree SST necessarily apply to this analysis.

This article is protected by copyright. All rights reserved. 


\section{Conclusion}

The presence of SVT and VOS discriminators in the setting of modern day realworld ICD programming plays a significant role in reducing current rates of inappropriate ICD therapies. In a simulated scenario without SVT and VOS discriminators on, the incidence of inappropriate therapies would have increased by $524 \%$ when compared to the actual study findings.

\section{Funding}

The PainFree SST study (URL: http://clinicaltrials.gov Unique Identifier: NCT00982397) was funded by Medtronic, plc.

Disclosures: AC, BG, MLB, DHF, DRL are employees and shareholders of Medtronic. AA: Consultant: Biosense Webster, Boston Scientific, Medtronic, and Microport CRM; Intellectual property: Biosense Webster, Boston Scientific, and Microport CRM; Speaker fees: Boston Scientific, Medtronic, Microport CRM, and Philips. EJS:

Honoraria/Speaking/Consulting Fee - Medtronic, Inc.; Boston Scientific Corp. Stock or Stock Options - Non-Public (Including Start-up) - AliveCor. TK: Speaker fees from Medtronic, Biotronik, Boston Scientific, and St. Jude Medical. LDS: Honoraria/Speaking/Consulting Fee - Bayer / Schering Pharma. KAE: Honorarium: Medtronic, Boston Scientific, Biotronik, Abbott; Research: Medtronic, Boston Scientific, Abbott; DSMB, Lectures, Consulting; Medtronic, Boston Scientific, Abbott, Biotronik. 


\section{References}

[1] Wilkoff BL, Fauchier L, Stiles MK, Morillo CA, Al-Khatib SM, Almendral J, Aguinaga L, Berger RD, Cuesta A, Daubert JP, Dubner S, Ellenbogen KA, Mark Estes NA, 3rd, Fenelon G, Garcia FC, Gasparini M, Haines DE, Healey JS, Hurtwitz JL, Keegan R, Kolb C, Kuck KH, Marinskis G, Martinelli M, McGuire M, Molina LG, Okumura K, Proclemer A, Russo AM, Singh JP, Swerdlow CD, Teo WS, Uribe W, Viskin S, Wang CC, Zhang S: 2015 HRS/EHRA/APHRS/SOLAECE expert consensus statement on optimal implantable cardioverter-defibrillator programming and testing. Heart Rhythm 2016; 13:e50-86.

[2] Wilkoff BL, Williamson BD, Stern RS, Moore SL, Lu F, Lee SW, Birgersdotter-Green UM, Wathen MS, Van Gelder IC, Heubner BM, Brown ML, Holloman KK, PREPARE Study Investigators: Strategic programming of detection and therapy parameters in implantable cardioverter-defibrillators reduces shocks in primary prevention patients: results from the PREPARE (Primary Prevention Parameters Evaluation) study. J Am Coll Cardiol 2008; 52:541-550.

[3] Gasparini M, Proclemer A, Klersy C, Kloppe A, Lunati M, Ferrer JB, Hersi A, Gulaj M, Wijfels MC, Santi E, Manotta L, Arenal A: Effect of long-detection interval vs standard-detection interval for implantable cardioverter-defibrillators on antitachycardia pacing and shock delivery: the ADVANCE III randomized clinical trial. JAMA 2013; 309:1903-1911.

[4] Moss AJ, Schuger C, Beck CA, Brown MW, Cannom DS, Daubert JP, Estes NA, 3rd, Greenberg H, Hall WJ, Huang DT, Kautzner J, Klein H, McNitt S, Olshansky 
B, Shoda M, Wilber D, Zareba W, MADIT-RIT Investigators: Reduction in inappropriate therapy and mortality through ICD programming. N Engl J Med 2012; 367:2275-2283.

[5] Wathen MS, DeGroot PJ, Sweeney MO, Stark AJ, Otterness MF, Adkisson WO, Canby RC, Khalighi K, Machado C, Rubenstein DS, Volosin KJ, Pain FREE Rx II Investigators: Prospective randomized multicenter trial of empirical antitachycardia pacing versus shocks for spontaneous rapid ventricular tachycardia in patients with implantable cardioverter-defibrillators: Pacing Fast Ventricular Tachycardia Reduces Shock Therapies (PainFREE Rx II) trial results. Circulation 2004; 110:2591-2596.

[6] Stiles MK, Fauchier L, Morillo CA, Wilkoff BL: 2019

HRS/EHRA/APHRS/LAHRS focused update to 2015 expert consensus statement on optimal implantable cardioverter-defibrillator programming and testing. Heart Rhythm 2019.

[7] Auricchio A, Meijer A, Kurita T, Schloss E, Brinkman K, Claessens-van

Ooijen M, Sterns L: Safety, efficacy, and performance of new discrimination algorithms to reduce inappropriate and unnecessary shocks: the PainFree SST clinical study design. Europace 2011; 13:1484-1493. Auricchio A, Schloss EJ, Kurita T, Meijer A, Gerritse B, Zweibel S, AlSmadi FM, Leng CT, Sterns LD, PainFree SST Investigators: Low inappropriate shock rates in patients with single- and dual/triple-chamber implantable cardioverter-defibrillators using a novel suite of detection algorithms: PainFree SST trial primary results. Heart Rhythm 2015; 12:926-936.

This article is protected by copyright. All rights reserved. 
DH, Rogers T: Inappropriate shocks in single-chamber and subcutaneous implantable cardioverter-defibrillators: a systematic review and meta-analysis. Europace 2017; 19:1973-1980.

[10] Sterns LD, Meine M, Kurita T, Meijer A, Auricchio A, Ando K, Leng CT, Okumura K, Sapp JL, Brown ML, Lexcen DR, Gerritse B, Schloss EJ: Extended detection time to reduce shocks is safe in secondary prevention patients: The secondary prevention substudy of PainFree SST. Heart Rhythm 2016; 13:1489-1496.

[11] Le-Rademacher J, Brazauskas R: Inference for Paired Survival Data. In Klein JP, van Houwelingen HC, Ibrahim JG, Scheike TH, eds: Handbook of Survival Analysis. Boca Raton, Florida USA: CRC Press, 2014, pp. 615-632.

[12] Gold MR, Ahmad S, Browne K, Berg KC, Thackeray L, Berger RD: Prospective comparison of discrimination algorithms to prevent inappropriate ICD therapy: primary results of the Rhythm ID Going Head to Head Trial. Heart Rhythm 2012; 9:370-377.

[13] Volosin KJ, Exner DV, Wathen MS, Sherfesee L, Scinicariello AP, Gillberg JM: Combining shock reduction strategies to enhance ICD therapy: a role for computer modeling. J Cardiovasc Electrophysiol 2011; 22:280-289.

[14] Swerdlow CD, Brown ML, Bordachar P: Sensing and detection with cardiac implantable electronic devices. Clinical Cardiac Pacing, Defibrillation and Resynchronization Therapy: Elsevier, 2017, pp. 114-167. 


\section{Figures}

Figure 1. Simulation scenarios. Panel A is an atrial fibrillation (AF) episode recorded during the PainFree SST trial. The patient was a 65 year old male with a history of AF, coronary artery disease, hypertension, and myocardial infarction. The AF detection rule of PR Logic, a component of the SST algorithms, begins withholding detection when the VT Count reaches VTNID (=16) indicated by AF in the decision channel as shown at the bottom right in the panel. Panel $\mathrm{B}$ is the same AF episode edited to provide a visual of the first simulation in which the SVT discriminators are off and other detection settings are unchanged. In this case, the episode would be detected when the VT Count reaches VTNID, and ATP therapy initiated as shown in the panel. Panel C illustrates the second simulation method. In this case, the same AF episode from panel A is simulated with SVT discriminators off and delayed high rate detection settings used. In this case, none of the beats are faster than the detection interval of $320 \mathrm{~ms}$ so the counter will not increment and the episode is not detected. Detailed description of the AF SVT algorithm has previously been reported. ${ }^{14}$ 

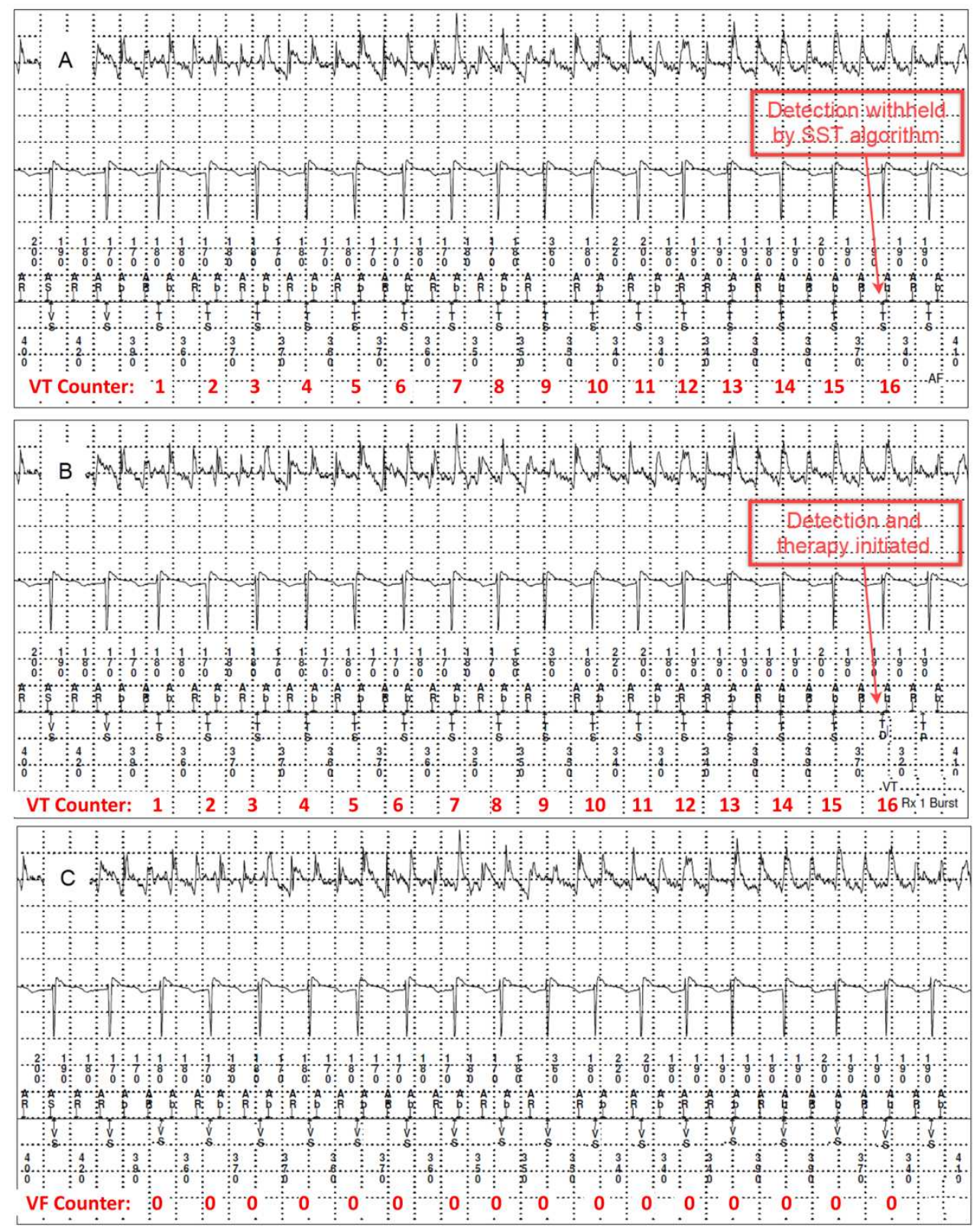

This article is protected by copyright. All rights reserved. 
Figure 2. Episode detection versus adjudicated rhythm. This includes all episodes with stored EGMs and at least 16 beats in the programmed detection zones. Detection withheld indicates which component of the SST algorithm withheld therapy.

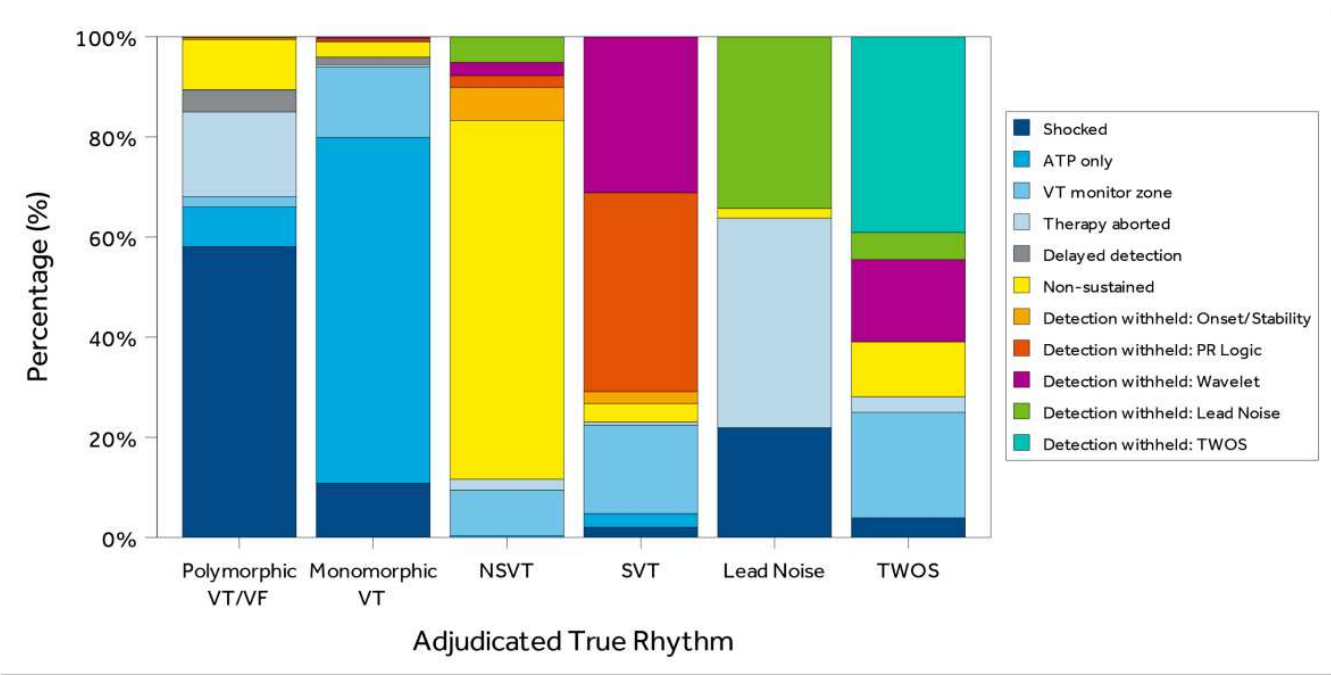

This article is protected by copyright. All rights reserved. 
Figure 3. Actual vs simulated inappropriate therapy rates. The figure includes all episodes with stored EGMs that met programmed detection criteria in the VT or VF zones, and either received inappropriate therapy or were appropriately classified as SVT by the device. The blue line shows actual therapy delivered with SVT discriminators on and detection criteria as programmed (PainFree SST study results). The red line is the simulated therapy that would have been delivered had the SVT discriminators been turned off and detection criteria as programmed. The green line shows the simulated therapy had SVT discriminators been turned off and programmed detection changed to having only a VF zone with NID 30/40 detection and a 320ms detection rate. The black line shows the simulated therapy had SVT discriminators been turned on and programmed detection changed to having only a VF zone with NID 30/40 detection and a $320 \mathrm{~ms}$ detection rate, which results in a $18.6 \%$ reduction of the inappropriate therapy rate compared to the actual incidence (blue line) $\mathrm{HR}=0.814$, not annotated on figure.

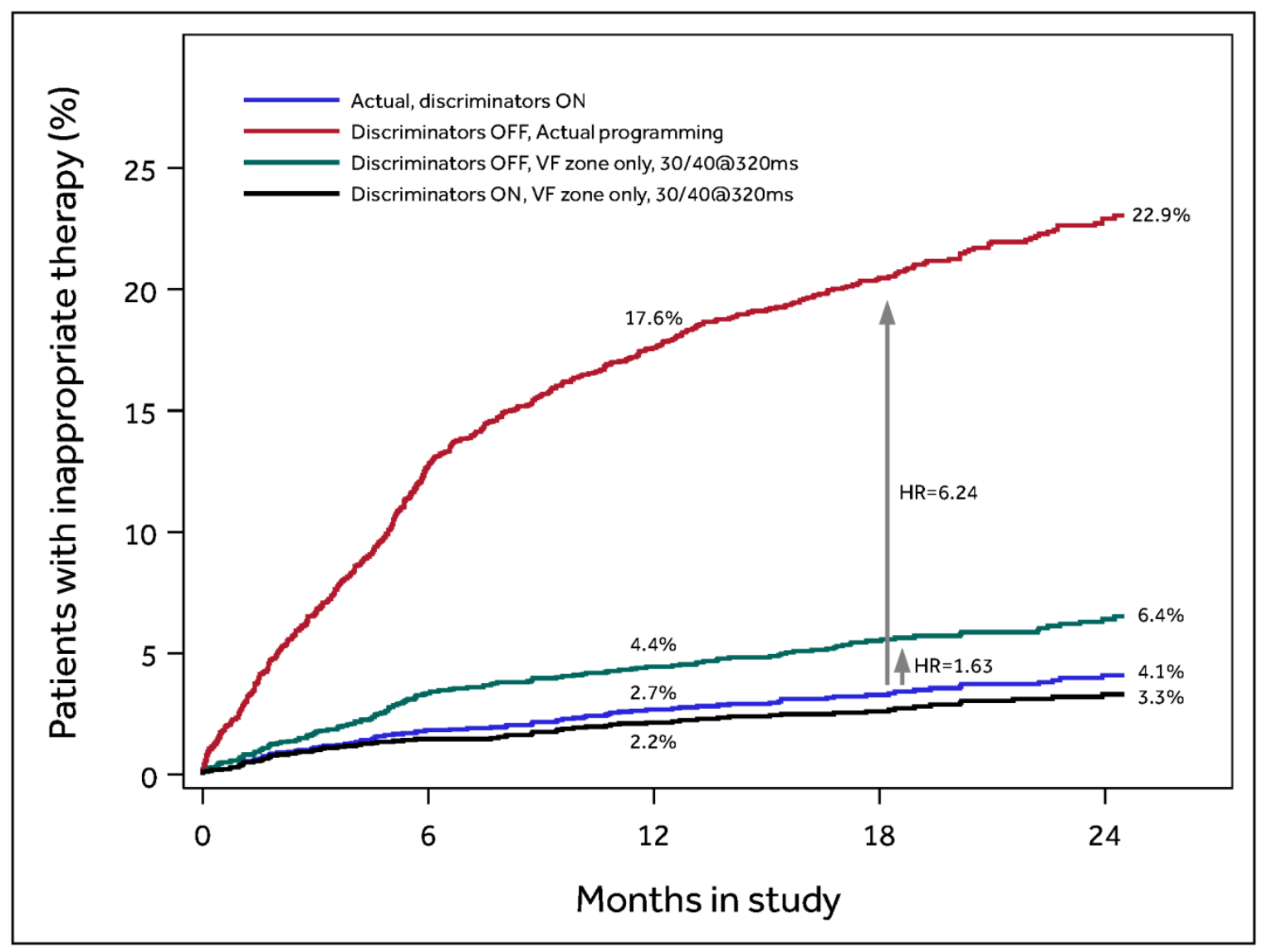

This article is protected by copyright. All rights reserved. 
Table 1: Patient characteristics

\begin{tabular}{|c|c|}
\hline Patient characteristics & $\begin{array}{l}\text { All patients } \\
(\mathrm{N}=\mathbf{2 7 7 0})\end{array}$ \\
\hline \multicolumn{2}{|l|}{ Demographics and Clinical Presentation } \\
\hline Male (N, \%) & $2200(79 \%)$ \\
\hline Age (years) & $65 \pm 12$ \\
\hline LVEF (\%) & $32 \pm 13$ \\
\hline QRS duration (msec) & $126 \pm 33$ \\
\hline Secondary prevention ( $N, \%)$ & $847(31 \%)$ \\
\hline \multicolumn{2}{|l|}{ NYHA class $(\mathrm{N}, \%)$} \\
\hline 1 & $419(15 \%)$ \\
\hline ॥ & $1104(40 \%)$ \\
\hline III & $853(31 \%)$ \\
\hline IV & $38(1 \%)$ \\
\hline No Heart Failure & $354(13 \%)$ \\
\hline \multicolumn{2}{|l|}{ History (N, \%) } \\
\hline Ischemic cardiomyopathy & $1206(44 \%)$ \\
\hline Coronary artery disease & $1745(63 \%)$ \\
\hline Previous device, any & $923(33 \%)$ \\
\hline \multicolumn{2}{|l|}{$\begin{array}{l}\text { Arrhythmias and Conduction Defects (N, } \\
\%)\end{array}$} \\
\hline Atrial fibrillation & $818(30 \%)$ \\
\hline Non-sustained Ventricular Tachycardia & $588(21 \%)$ \\
\hline $\begin{array}{l}\text { Sustained monomorphic Ventricular } \\
\text { Tachycardia }\end{array}$ & $281(10 \%)$ \\
\hline AV block & $404(15 \%)$ \\
\hline Left bundle branch block & $699(25 \%)$ \\
\hline Right bundle branch block & $215(8 \%)$ \\
\hline
\end{tabular}

This article is protected by copyright. All rights reserved. 


\begin{tabular}{|c|c|c|c|c|}
\hline Patient characteristics & \multicolumn{2}{|c|}{$\begin{array}{l}\text { All patients } \\
(\mathrm{N}=\mathbf{2 7 7 0})\end{array}$} & & \\
\hline \multicolumn{5}{|l|}{ Device (N, \%) } \\
\hline CRT-D & \multicolumn{2}{|c|}{$1071(39 \%)$} & & \\
\hline Dual-chamber ICD & \multicolumn{2}{|c|}{$948(34 \%)$} & & \\
\hline Single-chamber ICD & \multicolumn{2}{|c|}{$751(27 \%)$} & & \\
\hline \multicolumn{5}{|c|}{$\begin{array}{l}\text { AV, Atrioventricular; CRT-D, Cardiac Resynchror } \\
\text { NYHA, New York Heart Association; ICD, Implan } \\
\text { Table 2: Detection programming }\end{array}$} \\
\hline Detection programming & $\begin{array}{l}\text { All patients } \\
(\mathrm{N}=\mathbf{2 7 7 0})\end{array}$ & $\begin{array}{l}\text { Primary } \\
\text { prevention }^{1} \\
(\mathbf{N}=1917)\end{array}$ & $\begin{array}{l}\text { Secondary } \\
\text { prevention }^{1} \\
(\mathbf{N}=\mathbf{8 4 7})\end{array}$ & value $^{2}$ \\
\hline $\begin{array}{l}\text { No programming details } \\
\text { available }\end{array}$ & $171(6.2 \%)$ & $138(7.2 \%)$ & $31(3.7 \%)$ & $\begin{array}{c}< \\
0.001\end{array}$ \\
\hline \multicolumn{5}{|l|}{ Detection programming } \\
\hline VF detection On & $2591(99.7 \%)$ & $1773(99.7 \%)$ & $814(99.8 \%)$ & 1.00 \\
\hline \multicolumn{5}{|l|}{ VF NID ${ }^{3}$} \\
\hline $18 / 24$ & 491 (19.0\%) & 69 (3.9\%) & $422(51.8 \%)$ & \\
\hline $30 / 40$ & $2084(80.4 \%)$ & $1693(95.5 \%)$ & $387(47.5 \%)$ & \\
\hline Other & $16(0.6 \%)$ & $11(0.6 \%)$ & $5(0.6 \%)$ & \\
\hline VF detection interval (ms) ${ }^{3}$ & $304 \pm 17$ & $303 \pm 17$ & $304 \pm 18$ & 0.31 \\
\hline FVT detection zone enabled & $880(33.9 \%)$ & $538(30.2 \%)$ & $341(41.8 \%)$ & $\begin{array}{c}< \\
0.0001\end{array}$ \\
\hline VT detection On & $1636(62.9 \%)$ & $1042(58.6 \%)$ & $592(72.5 \%)$ & $\begin{array}{c}< \\
0.0001\end{array}$ \\
\hline
\end{tabular}

This article is protected by copyright. All rights reserved. 


\begin{tabular}{|c|c|c|c|c|}
\hline Detection programming & $\begin{array}{l}\text { All patients } \\
(\mathrm{N}=\mathbf{2 7 7 0})\end{array}$ & $\begin{array}{c}\text { Primary } \\
\text { prevention }^{1} \\
(\mathbf{N}=1917)\end{array}$ & $\begin{array}{l}\text { Secondary } \\
\text { prevention }^{1} \\
(\mathrm{~N}=\mathbf{8 4 7})\end{array}$ & $\begin{array}{c}\text { P- } \\
\text { value }^{2}\end{array}$ \\
\hline VT NID ${ }^{3}$ & $21 \pm 5$ & $21 \pm 5$ & $21 \pm 6$ & 0.86 \\
\hline VT interval $(\mathrm{ms})^{3}$ & $357 \pm 30$ & $350 \pm 21$ & $370 \pm 38$ & $\begin{array}{c}< \\
0.0001\end{array}$ \\
\hline Any VT therapy & $1610(61.9 \%)$ & 1022 (57.4\%) & $586(71.8 \%)$ & $\begin{array}{c}< \\
0.0001\end{array}$ \\
\hline Any VT shock therapy & $1545(59.4 \%)$ & 996 (56.0\%) & $547(67.0 \%)$ & $\begin{array}{c}< \\
0.0001\end{array}$ \\
\hline \multicolumn{5}{|l|}{$\begin{array}{l}\text { SVT discrimination enabled } \\
(\mathrm{N}, \%)\end{array}$} \\
\hline$A F$ rejection rule enabled ${ }^{4}$ & $1831(97.1 \%)$ & $1284(97.6 \%)$ & $545(95.8 \%)$ & 0.036 \\
\hline $\begin{array}{l}\text { Sinus tachycardia rejection rule } \\
\text { enabled }^{4}\end{array}$ & $1831(97.1 \%)$ & $1284(97.6 \%)$ & $545(95.8 \%)$ & 0.036 \\
\hline Wavelet rejection rule enabled & $2531(97.4 \%)$ & $1742(97.9 \%)$ & $785(96.2 \%)$ & 0.024 \\
\hline All enabled & 2485 (95.6\%) & $1713(96.3 \%)$ & $768(94.1 \%)$ & 0.017 \\
\hline $\begin{array}{l}\text { SVT limit programmed nominal } \\
(260 \mathrm{~ms})\end{array}$ & $2544(97.9 \%)$ & $1740(97.8 \%)$ & $800(98.0 \%)$ & 0.77 \\
\hline
\end{tabular}

CRT-D, Cardiac Resynchronization Therapy Defibrillator; FVT, Fast Ventricular Tachycardia; ms, millisecond; NID, Number of Intervals to Detect; SVT, Supraventricular Tachycardia; VF, Ventricular Fibrillation; VT, Ventricular Tachycardia

${ }^{1}$ Indication is missing for 6 patients

${ }^{2}$ Tests are Student's t-test for continuous and Fisher's exact test for categorical variables

${ }^{3}$ Includes only the cases where the respective detection zone is enabled

${ }^{4}$ Not available in Single-Chamber devices

This article is protected by copyright. All rights reserved. 
Table 3: Episode rhythm vs adjudicated rhythm. This includes all 9,660 episodes with stored EGM and at least 16 beats in the programmed detection zones. There were 10 true VT/VF episodes that received an inappropriate shock after spontaneous termination and 4 SVT/VOS episodes that received an appropriate shock after inappropriate ATP. The 3,282 SVT/VOS episodes that met the ratebased detection criteria are the episodes with inappropriate therapy in the top two rows and the episodes with detection withheld in the bottom 5 rows.

\begin{tabular}{|c|c|c|c|c|c|c|}
\hline Detection & $\begin{array}{c}\text { Polymorphic } \\
\text { VT/VF } \\
(\mathbf{N}=\mathbf{3 7 9})\end{array}$ & $\begin{array}{c}\text { Monomorphic } \\
\text { VT } \\
(\mathbf{N}=\mathbf{4 3 8 9})\end{array}$ & $\begin{array}{c}\text { NSVT } \\
(\mathbf{N}=645)\end{array}$ & $\begin{array}{c}\text { SVT } \\
(\mathrm{N}=\mathbf{4 0 1 4})\end{array}$ & $\begin{array}{c}\text { Lead Noise } \\
(\mathrm{N}=\mathbf{1 0 5})\end{array}$ & $\begin{array}{c}\text { TWOS } \\
(\mathrm{N}=\mathbf{1 2 8})\end{array}$ \\
\hline Shocked & $220(58.0 \%)$ & $475(10.8 \%)$ & $0(0.0 \%)$ & $81(2.0 \%)$ & $23(21.9 \%)$ & $5(3.9 \%)$ \\
\hline ATP only & $30(7.9 \%)$ & $3031(69.1 \%)$ & $2(0.3 \%)$ & $110(2.7 \%)$ & $0(0.0 \%)$ & $0(0.0 \%)$ \\
\hline VT monitor zone & $8(2.1 \%)$ & $619(14.1 \%)$ & $59(9.1 \%)$ & $710(17.7 \%)$ & $0(0.0 \%)$ & $27(21.1 \%)$ \\
\hline Therapy aborted & $64(16.9 \%)$ & $21(0.5 \%)$ & $14(2.2 \%)$ & $24(0.6 \%)$ & $44(41.9 \%)$ & $4(3.1 \%)$ \\
\hline Delayed detection & $17(4.5 \%)$ & $65(1.5 \%)$ & $0(0.0 \%)$ & $0(0.0 \%)$ & $0(0.0 \%)$ & $0(0.0 \%)$ \\
\hline Non-sustained & $38(10.0 \%)$ & $132(3.0 \%)$ & $462(71.6 \%)$ & $149(3.7 \%)$ & $2(1.9 \%)$ & $14(10.9 \%)$ \\
\hline Detection withheld: Onset/Stability & $1(0.3 \%)$ & $10(0.2 \%)$ & $43(6.7 \%)$ & $95(2.4 \%)$ & $0(0.0 \%)$ & $0(0.0 \%)$ \\
\hline Detection withheld: PR Logic & $1(0.3 \%)$ & $21(0.5 \%)$ & $15(2.3 \%)$ & $1595(39.7 \%)$ & $0(0.0 \%)$ & $0(0.0 \%)$ \\
\hline Detection withheld: Wavelet & $0(0.0 \%)$ & $15(0.3 \%)$ & $17(2.6 \%)$ & $1250(31.1 \%)$ & $0(0.0 \%)$ & $21(16.4 \%)$ \\
\hline Detection withheld: Lead Noise & $0(0.0 \%)$ & $0(0.0 \%)$ & $33(5.1 \%)$ & $0(0.0 \%)$ & $36(34.3 \%)$ & $7(5.5 \%)$ \\
\hline Detection withheld: TWOS & $0(0.0 \%)$ & $0(0.0 \%)$ & $0(0.0 \%)$ & $0(0.0 \%)$ & $0(0.0 \%)$ & $50(39.1 \%)$ \\
\hline
\end{tabular}

ATP, Antitachycardia Pacing; NSVT, Non-Sustained Ventricular Tachycardia; SVT, Supraventricular Tachycardia; TWOS, T-wave Oversensing; VF, Ventricular Fibrillation; VT, Ventricular Tachycardia

This article is protected by copyright. All rights reserved. 